\title{
A Modified Arthroscopic All-inside Repair for Lateral Meniscal Tears Anterior to the Popliteal Hiatus: A Retrospective Case Series with Minimum 2-Year Follow-Up
}

Hongwu Zhuo ( $\nabla$ zhuohongwu2019@163.com )

Fuzhou Second Hospital Affiliated to Xiamen University

Fugui Zhu

fuzhou second hospital affiliated to xiamen university

Ling Pan

fuzhou second hospital affiliated to xiamen university

Jian Li

Fuzhou Second Hospital Affiliated to Xiamen University

Research article

Keywords: arthroscopy, all-inside repair, lateral meniscus, popliteal hiatus

Posted Date: April 21st, 2020

DOI: https://doi.org/10.21203/rs.3.rs-21032/v1

License: (c) (1) This work is licensed under a Creative Commons Attribution 4.0 International License.

Read Full License 


\section{Abstract}

\section{Background}

To determine the clinical and MRI outcomes after modified arthroscopic all-inside repair for lateral meniscal tears anterior to the popliteal hiatus.

\section{Methods}

Patients who underwent modified arthroscopic all-inside repair for lateral meniscal tears anterior to the popliteal hiatus at our institution were identified. The clinical assessment consisted of the symptoms of meniscal tears, McMurray test, and patient-reported outcomes (Lysholm score, Tegner score, and International Knee Documentation Committee (IKDC) score). The status of meniscal healing was assessed using postoperative MRI scan.

\section{Results}

Twenty-five patients met the inclusion criteria. The mean age was $27.60 \pm 8.37$ years (range, 16 to 43 years). The mean follow-up period was $26.04 \pm 2.88$ months (range, 24 to 36 months). At final follow-up, the symptoms of meniscal tears disappeared in 23 patients with a negative McMurray test. The patientreported outcomes of Lysholm, Tegner, and IKDC score improved significantly compared to the preoperative values $(P=0.001)$. No significant differences were observed in postoperative clinical outcomes between patients with or without concomitant ACL tears $(P>0.05)$. Postoperative MRI scan showed that the repaired lateral meniscus anterior to the popliteal hiatus obtained healing in $23(92.0 \%)$ patients and no healing in 2 patients.

\section{Conclusion}

This modified arthroscopic all-inside repair technique was safe and effective to treat lateral meniscal tears anterior to the popliteal hiatus, resulting in significantly improved clinical outcomes with a high healing rate and low risk of adjacent structures injury.

\section{Background}

Over the past decades, the anatomy and function of the lateral meniscus have been well recognized. The arthroscopic techniques for lateral meniscal repair have also evolved dramatically [1, 2]. However, arthroscopic repair for lateral meniscal tears anterior to the popliteal hiatus still presents a clinical challenge for orthopedic surgeons. The reason for this is multifactorial. First, several important structures are immediately behind the lateral meniscus, such as popliteus tendon, inferior lateral genicular artery (ILGA), and common peroneal nerve. There is a consensus regarding the possibility of iatrogenic injury to these adjacent structures during surgical procedure [3-7]. Recently, Uchida et al. reported that the rate of popliteus tendon injury could be as high as $94 \%$ during lateral meniscal repair anterior to the popliteal hiatus using FasT-Fix system [7]. Secondly, the joint capsule around the popliteal hiatus is quite thin. 
Therefore, suture knots or anchors could not be placed securely over the capsule [3]. Thirdly, the connection between the lateral meniscus and the joint capsule in this region is interrupted by popliteal hiatus, resulting in poor vascular supply and low healing rate. In a previous study, Horibe et al. demonstrated that the lateral meniscus anterior to the popliteal hiatus had a significantly lower healing rate compared with lateral meniscal posterior horn $(63 \%$ vs. $97 \%, P<0.05)$ [8]. Therefore, how to safely and effectively repair the lateral meniscal tears anterior to the popliteal hiatus and promote the healing are the main challenges in treating these lesions.

Although lateral meniscal tears anterior to the popliteal hiatus is quite common in clinical practice, to our best knowledge, there is still very limited data in the literature regarding optimal arthroscopic repair technique for these lesions [9-11]. Since July 2015, we started to perform a modified arthroscopic allinside repair using spinal needle and suture hook for lateral meniscal tears anterior to the popliteal hiatus. This study aimed to determine the clinical and MRI outcomes after this procedure. We hypothesized that this procedure would yield satisfactory clinical and MRI outcomes with a high healing rate and low risk of adjacent structures injury.

\section{Methods}

\section{Study design}

From July 2015 to December 2017, the medical records of patients who underwent modified arthroscopic all-inside repair for lateral meniscal tears anterior to the popliteal hiatus at our institution were identified. The indication of this procedure was a lateral meniscal tear anterior to the popliteal hiatus which was fullthickness or nearly full-thickness, longitudinal, with a length of tear $>1 \mathrm{~cm}$, and located in red-red zone or red-white zone. The inclusion criteria were (1) patients who were diagnosed with lateral meniscal tears anterior to the popliteal hiatus and underwent modified arthroscopic all-inside repair, and (2) patients with a minimum 2-year follow-up. The exclusion criteria were (1) age older than 45 years, (2) patients with concomitant medial meniscal tears, (3) significant osteoarthritis of the joint (Kellgren-Lawrence grade III or IV), or (4) previous surgery of the same knee.

A total of 39 patients (39 knees) were identified (Fig. 1). As 12 patients had concomitant medial meniscal tears, 1 patient had previous surgery and another patient was lost to follow-up, we studied the remaining 25 patients ( 25 knees). Among them, 16 patients had concomitant anterior cruciate ligament (ACL) tear. The remaining 9 patients were isolated lateral meniscal tear. Preoperatively, all patients had different degrees of symptoms of meniscal tears, including pain, effusion, snapping and catching. And a positive McMurray test was identified in all patients.

This study received approval from our institutional review board. Informed consent was obtained from all individual participants included in the study.

\section{Surgical technique}


All surgeries were performed under general anesthesia. The patients were placed in a supine position. A routine arthroscopic evaluation was conducted using two standard anterior knee arthroscopy portals. If the ACL was torn, the lateral meniscus was addressed before ACL reconstruction.

Once a lateral meniscal tear anterior to the popliteal hiatus was confirmed (Fig. 2a), the torn edge was refreshed using a meniscal rasp. An 18-gauge spinal needle could also be utilized to pierce the torn edge to encourage bleeding.

Then the arthroscopy was placed in anterolateral portal, and the anteromedial portal was used as working portal. Passed an 18-gauge spinal needle preloaded with a No. 2 polydioxanone suture (PDS; Ethicon, Somerville, $\mathrm{NJ}$ ) through the skin approximately $1.5 \sim 2 \mathrm{~cm}$ anterosuperior to the tip of the fibular head, subcutaneous tissue, and capsule. The needle emerged from the inferior surface of the lateral meniscus. Advanced the PDS suture through the spinal needle and the end of PDS suture was pulled out via the anteromedial portal using a suture retriever (Fig. 2b). Pulled back the needle out of the skin and the other end of PDS suture was pulled out over the superior surface of the lateral meniscus.

A $45^{\circ}$ suture hook preloaded with a suture loop was introduced via the anteromedial portal and pierced through the inner part of the meniscus approximately $5 \mathrm{~mm}$ away from the torn edge. The suture loop was used as a shuttle relay to transport the end of PDS suture (on the inferior surface of the lateral meniscus) to the superior surface of the lateral meniscus. Now both the ends of PDS suture were on the superior surface of the lateral meniscus and became a vertical mattress stitch ready to be tied (Fig. 2c). Tied the sutures with a knot-pusher and adjusted the knot into the popliteal hiatus to avoid knot impingement with articular cartilage (Fig. 2d). The same maneuver was repeated as necessary to provide a stable meniscal repair. According to our experience, two stitches were usually needed for lateral meniscal tears anterior to the popliteal hiatus. If the meniscal tears extended anteriorly to the meniscal body or posteriorly to the posterior horn, the extension part was repaired using FasT-Fix system (Smith \& Nephew, USA). After completion of lateral meniscal repair, the suture tension was checked with a probe.

\section{Postoperative rehabilitation}

Postoperatively, a hinged brace was applied for 12 weeks. Quadricep-strengthening exercises were started on the second day. Flexion was limited to 90 degrees during the first 4 weeks and gradually increased to normal at 12 weeks. Toe-touch weight-bearing was permitted at 4 weeks and full weight-bearing with the brace was permitted at 6 weeks. Squatting and swimming were started at 3 months. Running and bicycling were started at 6 months. Full return to competitive sports was allowed at 12 months.

\section{Assessment}

Demographic data were collected from the database, including age, sex, side of injury, time from injury to surgery, and concomitant surgical procedures. Clinical outcomes were assessed preoperatively and at final follow-up. 
The clinical outcomes were assessed based on (1) symptoms of meniscal tears, including pain, effusion, snapping and catching, (2) McMurray test, and (3) patient-reported outcomes (Lysholm score, Tegner score, and International Knee Documentation Committee (IKDC) score). For the patients undergoing concomitant ACL reconstruction, the KT-1000 arthrometer (Metric, San Diego, USA) was used to measure the side-to-side difference (SSD) for assessment of joint stability.

At final follow-up, all patients underwent MRI (3.0-T MR System, Signa Excite, GE Medical Systems, Waukesha, Wisconsin, USA) of the knee joint to assess the status of meniscal healing. According to Lim's criteria, if there was no high-signal-intensity linear area extending to an articular surface of the meniscus, it was regarded as meniscal healing. Otherwise, it was regarded as no healing [12].

\section{Statistical analysis}

All statistical analyses were performed using SPSS software (IBM-SPSS statistics 22.0; New York, USA). Continuous variables were presented as the mean \pm standard deviation. A paired-samples $t$ test was used to determine the differences between preoperative and postoperative quantitative variables. In order to assess the influence of concomitant ACL tears on postoperative patient-reported outcomes, an independent-samples $t$ test was used to determine the differences in postoperative clinical outcomes between patients with or without concomitant ACL tears. The significance level was set at 0.05 .

\section{Result}

\section{Demographic data}

In the end, 25 patients met the inclusion criteria and were enrolled in this study. There were 16 males and 9 females with a mean age of $27.60 \pm 8.37$ years (range, 16 to 43 years). Among this group, 16 patients underwent concomitant ACL reconstruction for ACL tear. The demographic data of the patients are summarized in Table 1.

\section{Table 1. Demographic data of the patients}

\begin{tabular}{ll} 
Variable & Value \\
\hline Patients, $\mathrm{n}$ & 25 \\
\hline Age, $\mathrm{y}$ & $27.60 \pm 8.37$ \\
\hline Sex, male/female & $16 / 9$ \\
\hline Side, right/left & $15 / 10$ \\
\hline Time from injury to surgery, d & $68.72 \pm 83.63$ \\
\hline Patients with concomitant ACL tear, $\mathrm{n}$ & 16 \\
\hline Follow-up period, mo & $26.04 \pm 2.88$
\end{tabular}


The data are shown as mean \pm standard deviation or $\mathrm{n}$. ACL, anterior cruciate ligament.

\section{Clinical outcomes}

The mean follow-up period was $26.04 \pm 2.88$ months (range, 24 to 36 months). At final follow-up, the symptoms of meniscal tears disappeared in 23 patients with a negative McMurray test. Two patients occasionally felt slight activity-related pain in the posterolateral area of the knee joint and a suspicious positive McMurray test was identified in both patients. For the patients with concomitant ACL reconstruction, the preoperative and postoperative SSD were $8.11 \pm 1.62 \mathrm{~mm}$ (range, 6 to $11 \mathrm{~mm}$ ) and $1.35 \pm 0.93 \mathrm{~mm}$ (range, 0 to $3 \mathrm{~mm}$ ), respectively. There was a significant difference between preoperative and postoperative SSD $(P=0.001)$.

The preoperative Lysholm score, Tegner score, and IKDC score were $41.44 \pm 17.66,1.89 \pm 1.15$, and 42.26 \pm 18.42 , respectively. The postoperative Lysholm score, Tegner score, and IKDC score were $99.60 \pm 1.38$, $6.16 \pm 0.89$, and $99.40 \pm 1.47$, respectively. There were significant differences between preoperative and postoperative patient-reported outcomes $(P=0.001)$ (Table 2).

Table 2. Comparison of the preoperative and postoperative patient-reported outcomes

\begin{tabular}{llll} 
Variables & Preoperative & Postoperative & P Value \\
\hline Lysholm score & $41.44 \pm 17.66$ & $99.60 \pm 1.38$ & .001 \\
\hline Tegner score & $1.89 \pm 1.15$ & $6.16 \pm 0.89$ & .001 \\
\hline IKDC score & $42.26 \pm 18.42$ & $99.40 \pm 1.47$ & .001
\end{tabular}

The data are shown as mean \pm standard deviation. IKDC score, International Knee Documentation Committee score.

The postoperative Lysholm score, Tegner score, and IKDC score in patients with concomitant ACL tear were $99.68 \pm 1.25,6.18 \pm 0.83$, and $99.43 \pm 1.24$, respectively, and in patients without concomitant ACL tear were $99.44 \pm 1.66,6.11 \pm 1.05$, and $99.36 \pm 1.90$, respectively. There were no significant differences in postoperative clinical outcomes between patients with or without concomitant ACL tears $(P>0.05)($ Table 3).

Table 3. Comparison of postoperative patient-reported outcomes between patients with or without concomitant ACL tears 


\begin{tabular}{lllc} 
Variables & $\begin{array}{l}\text { Patients with concomitant ACL } \\
\text { tear }(n=16)\end{array}$ & $\begin{array}{l}\text { Patients without concomitant } \\
\text { ACL tear }(n=9)\end{array}$ & $\begin{array}{c}\text { P } \\
\text { Value }\end{array}$ \\
\hline $\begin{array}{l}\text { Postoperative } \\
\text { Lysholm score }\end{array}$ & $99.68 \pm 1.25$ & $99.44 \pm 1.66$ & .683 \\
\hline $\begin{array}{l}\text { Postoperative Tegner } \\
\text { score }\end{array}$ & $6.18 \pm 0.83$ & $6.11 \pm 1.05$ & .843 \\
\hline $\begin{array}{l}\text { Postoperative IKDC } \\
\text { score }\end{array}$ & $99.43 \pm 1.24$ & $99.36 \pm 1.90$ & .927
\end{tabular}

The data are shown as mean \pm standard deviation. IKDC score, International Knee Documentation Committee score.

\section{MRI outcomes}

At final follow-up, postoperative MRI scan showed that the repaired lateral meniscus anterior to the popliteal hiatus obtained healing in 23 (92.0\%) patients and no healing in 2 patients (Fig. 3).

\section{Complications}

No infection, arthrofibrosis, or injury of adjacent structures (including popliteus tendon, ILGA, and common peroneal nerve) occurred among this group. Although two patients occasionally felt slight activity-related pain, no reoperation was required.

\section{Discussion}

The main finding of this study was that this modified arthroscopic all-inside repair for patients with lateral meniscal tears anterior to the popliteal hiatus yielded favorable clinical and MRI outcomes at a mean follow-up of 26.04 months. A high rate of meniscal healing (92.0\%) and low risk of adjacent structures injury were observed. In addition, patients with concomitant ACL tears had similar postoperative patient-reported outcomes compared to patients without concomitant ACL tears.

In 1979, Cohn was the first to report that the popliteal hiatus was a constant feature of normal knee anatomy, with an average length of $1.3 \mathrm{~cm}$ [13]. Similarly, Aman et al. described that the mean length of superior popliteal hiatus exceeded $12.1 \mathrm{~mm}$ and the mean length of inferior popliteal hiatus was up to $36.9 \mathrm{~mm}$ [3]. It is generally considered that a meniscal tear requires surgery for better stability if the length of tear exceeds $1 \mathrm{~cm}$ [1]. Therefore, when a lateral meniscal tear anterior to the popliteal hiatus occurs and exceeds $1 \mathrm{~cm}$, surgery should be considered. Currently, the surgical methods for meniscal tears mainly include meniscectomy and meniscal repair. As multiple studies have shown that meniscectomy would lead to decreased tibiofemoral joint contact area, increased cartilage stress load, and accelerate 
degenerative joint changes [14-16]. Therefore, meniscal repair should be considered a priority in managing meniscal tears, especially for young patients.

For lateral meniscal repair anterior to the popliteal hiatus, some authors suggested using the popliteus tendon for meniscal repair device placement, and preliminary data have been reported $[10,11]$.

Nevertheless, this technique has several disadvantages. First, it provides a non-anatomical repair, which would close down the popliteal hiatus, reduce the normal mobility of lateral meniscus, and even cause iatrogenic lateral meniscus extrusion [17-19]. Second, it has a high risk of adjacent structures injury, including popliteus tendon, ILGA, and common peroneal nerve [4-7]. The ILGA has been reported to be the main source of blood supply for lateral meniscus anterior to the popliteal hiatus, so its injury would significantly influence the meniscal healing [20]. Third, the suture anchors placed within or outside the popliteus tendon was a risk factor for local irritation, as well as suture loosening because of the anterior movement of the anchors in popliteus tendon during knee motion [21, 22].

Alternatively, Farrow et al. described placing the suture anchors just outside the lateral meniscus without penetrating the popliteus tendon [9]. This technique aimed to restore the native physiologic properties of the lateral meniscus and avoid injury to adjacent structures. However, this technique is more technically challenging as it requires an accurate depth of penetration to avoid overpenetration or underpenetration. Thus, it is not fit for the surgeons in early learning curve. Furthermore, it requires both sides of the torn edge have sufficient tissue, resulting in a limited clinical application.

In this study, we present a modified arthroscopic all-inside repair technique using spinal needle and suture hook for lateral meniscal tears anterior to the popliteal hiatus. Compared with other techniques, we believe our technique has some advantages. First, our technique provides an anatomical repair. Therefore, it preserves the native physiologic properties of the lateral meniscus. Second, all the manipulations are conducted before the adjacent structures and under direct arthroscopic visualization, resulting in a relatively low risk of adjacent structures injury. Third, our technique is less technically challenging and does not require specific repair instrumentation, so it is fit for surgeons in early learning curve.

\section{Limitations}

There are some limitations in this study. First, this study was a retrospective study with all the inherent limitations of a retrospective study. Second, this study did not involve any control group. Future blinded comparative study is required to determine whether our technique is superior to other repair techniques. Third, successful healing of the repaired lateral meniscus was not confirmed by second-look arthroscopy. Second-look arthroscopy allows for direct inspection of the repair site. However, since it is invasive and expensive, it is rarely performed at our institution.

\section{Conclusions}


Overall, this modified arthroscopic all-inside repair technique was safe and effective to treat lateral meniscal tears anterior to the popliteal hiatus, resulting in significantly improved clinical outcomes with a high healing rate and low risk of adjacent structures injury.

\section{Abbreviations}

IKDC: International Knee Documentation Committee

ILGA: inferior lateral genicular artery

ACL: Anterior cruciate ligament

PDS: polydioxanone suture

SSD: side-to-side difference

\section{Declarations}

\section{Ethics approval and consent to participate}

All patients provided signed informed consent to allow their clinical and radiologic data to be used for research programmes.

The name of the Board: Institutional Review Board of Fuzhou Second Hospital of Xiamen University. Approval number is INR 10020190112.

\section{Consent for publication}

All patients signed informed consent regarding publishing their data and photographs.

\section{Availability of data and materials}

The raw data are available from the corresponding author upon reasonable request.

\section{Competing interests}

The authors declare that they have no competing interests.

\section{Funding}

None.

\section{Authors' contributions}

All authors contributed to the study conception and design. $\mathrm{HZ}$ analysed and interpreted the patient data. FZ and LP were major contributors to manuscript writing. JL performed all the surgeries. All authors read 
and approved the final manuscript.

\section{Acknowledgements}

Not applicable.

\section{References}

1. Doral MN, Bilge O, Huri G et al Modern treatment of meniscal tears. EFORT Open Rev. 2018;3(5): 260268.

2. Spalding T, Damasena I, Lawton R Meniscal Repair Techniques. Clin Sports Med. 2020;39(1): 37-56.

3. Aman ZS, DePhillipo NN, Storaci HW et al Quantitative and Qualitative Assessment of Posterolateral Meniscal Anatomy: Defining the Popliteal Hiatus, Popliteomeniscal Fascicles, and the Lateral Meniscotibial Ligament. Am J Sport Med. 2019;47(8): 1797-1803.

4. Cuellar A, Cuellar R, Heredia JD et al The all-inside meniscal repair technique has less risk of injury to the lateral geniculate artery than the inside-out repair technique when suturing the lateral meniscus. Knee Surg Sport Tr A. 2018;26(3): 793-798.

5. Massey P, Parker D, Feibel B et al Proximity of the Neurovascular Bundle During Posterior-Lateral Meniscal Repair: A Comparison of the Transpatellar, Anteromedial, and Anterolateral Portals. Arthroscopy-the Journal of Arthroscopic and Related Surgery. 2019;35(5): 1557-1564.

6. McCulloch PC, Jones HL, Lue J et al What Is the Optimal Minimum Penetration Depth for "All-Inside" Meniscal Repairs? Arthroscopy. 2016;32(8): 1624-1630.

7. Uchida R, Mae T, Hiramatsu K et al Effects of suture site or penetration depth on anchor location in all-inside meniscal repair. Knee. 2016;23(6): 1024-1028.

8. Horibe S, Shino K, Nakata K et al Second-look arthroscopy after meniscal repair. Review of 132 menisci repaired by an arthroscopic inside-out technique. J Bone Joint Surg Br. 1995;77(2): 245-249.

9. Farrow L Arthroscopic Management of Meniscus Tears at the Popliteal Hiatus. Oper Techn Sport Med. 2018;26(4): 251-255.

10. Kang HJ, Chun $\mathrm{CH}$, Kim KM et al The Results of All-Inside Meniscus Repair Using the Viper Repair System Simultaneously with Anterior Cruciate Ligament Reconstruction. Clin Orthop Surg. 2015;7(2): 177-184.

11. Ouanezar H, Blakeney WG, Latrobe $\mathrm{C}$ et al The popliteus tendon provides a safe and reliable location for all-inside meniscal repair device placement. Knee Surg Sports Traumatol Arthrosc. 2018;26(12): 3611-3619.

12. Lim PS, Schweitzer ME, Bhatia $M$ et al Repeat tear of postoperative meniscus: Potential MR imaging signs. Radiology. 1999;210(1): 183-188.

13. Cohn AK, Mains DB Popliteal hiatus of the lateral meniscus. Anatomy and measurement at dissection of 10 specimens. Am J Sports Med. 1979;7(4): 221-226. 
14. Chahla J, Cinque ME, Godin JA et al Meniscectomy and Resultant Articular Cartilage Lesions of the Knee Among Prospective National Football League Players: An Imaging and Performance Analysis. Am J Sports Med. 2018;46(1): 200-207.

15. Lau BC, Conway D, Mulvihill J et al Biomechanical Consequences of Meniscal Tear, Partial Meniscectomy, and Meniscal Repair in the Knee. JBJS Rev. 2018;6(4): e3.

16. Willinger L, Foehr P, Achtnich A et al Effect of Lower Limb Alignment in Medial Meniscus-Deficient Knees on Tibiofemoral Contact Pressure. Orthop J Sports Med. 2019;7(2).

17. Bryceland JK, Powell AJ, Nunn T Knee Menisci: Structure, Function, and Management of Pathology. Cartilage. 2017;8(2): 99-104.

18. Kashihara N, Furumatsu T, Kodama $\mathrm{Y}$ et al Concurrent Lateral Meniscal Repair with Anterior Cruciate Ligament Reconstruction Induces the Extrusion of the Lateral Meniscus: Assessments of Magnetic Resonance Images. Acta Med Okayama. 2016;70(6): 441-448.

19. Zhang F, Bierma-Zeinstra SM, Oei EHG et al Factors associated with meniscal body extrusion on knee MRI in overweight and obese women. Osteoarthr Cartilage. 2017;25(5): 694-699.

20. Day B, Mackenzie WG, Shim SS et al The vascular and nerve supply of the human meniscus. Arthroscopy. 1985;1(1): 58-62.

21. Grant JA, Wilde J, Miller BS et al Comparison of Inside-Out and All-Inside Techniques for the Repair of Isolated Meniscal Tears A Systematic Review. Am J Sport Med. 2012;40(2): 459-468.

22. Warth LC, Bollier MJ, Hoffman DF et al New Complication Associated With All-Inside Meniscal Repair Device: Ultrasound-Aided Diagnosis and Operative Localization of Foreign Body Reaction. Orthop J Sports Med. 2016;4(9).

\section{Figures}




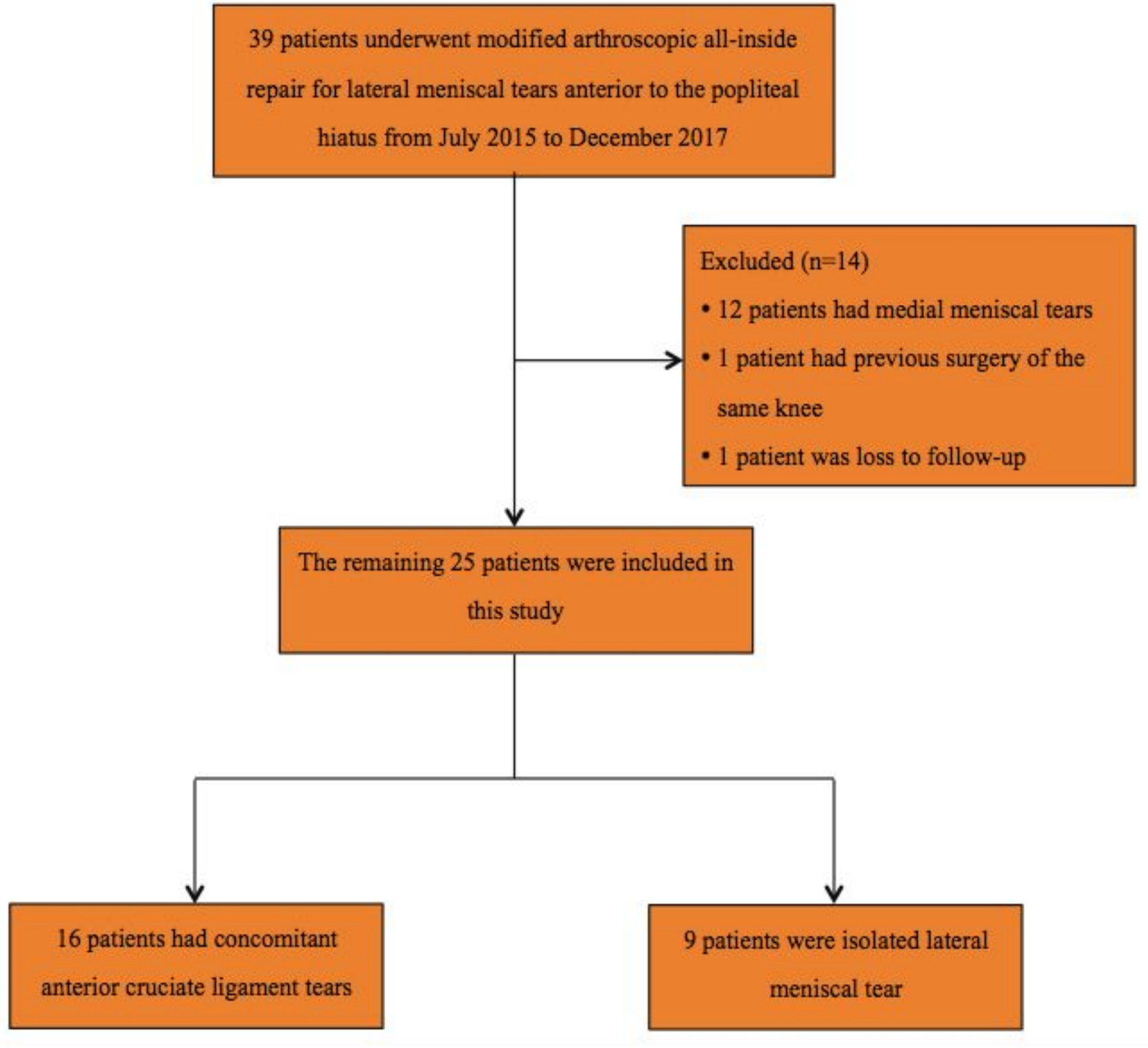

\section{Figure 1}

Flow chart of this study. 

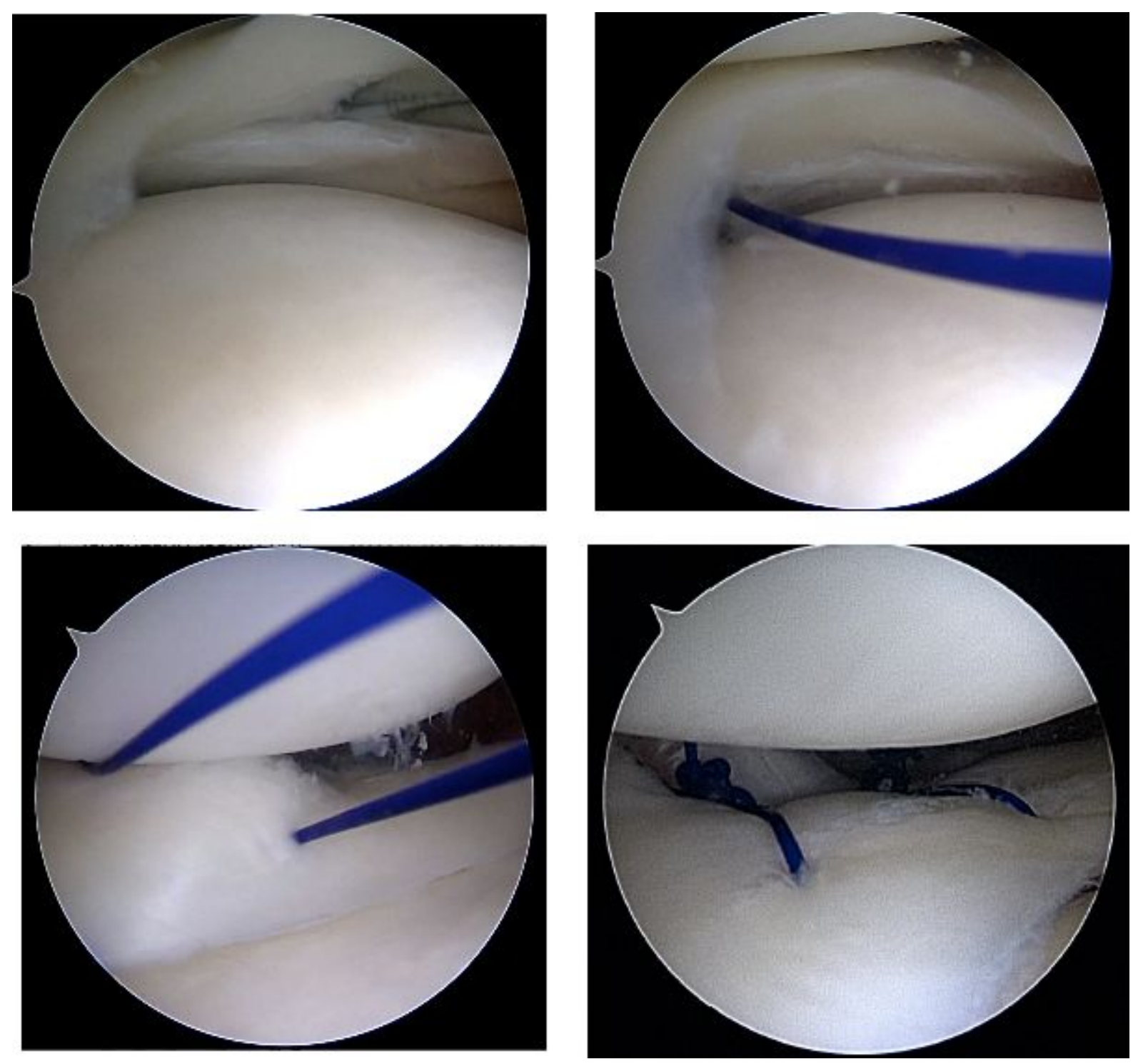

\section{Figure 2}

Modified arthroscopic all-inside repair for lateral meniscal tears anterior to the popliteal hiatus (right knee). a A lateral meniscal tear anterior to the popliteal hiatus was confirmed by a probe. b One end of PDS suture was pulled out from the inferior surface of the lateral meniscus. $c$ Both the ends of PDS suture were on the superior surface of the lateral meniscus and became a vertical mattress stitch ready to be tied. $d$ Adjusted the knot into the popliteal hiatus. 

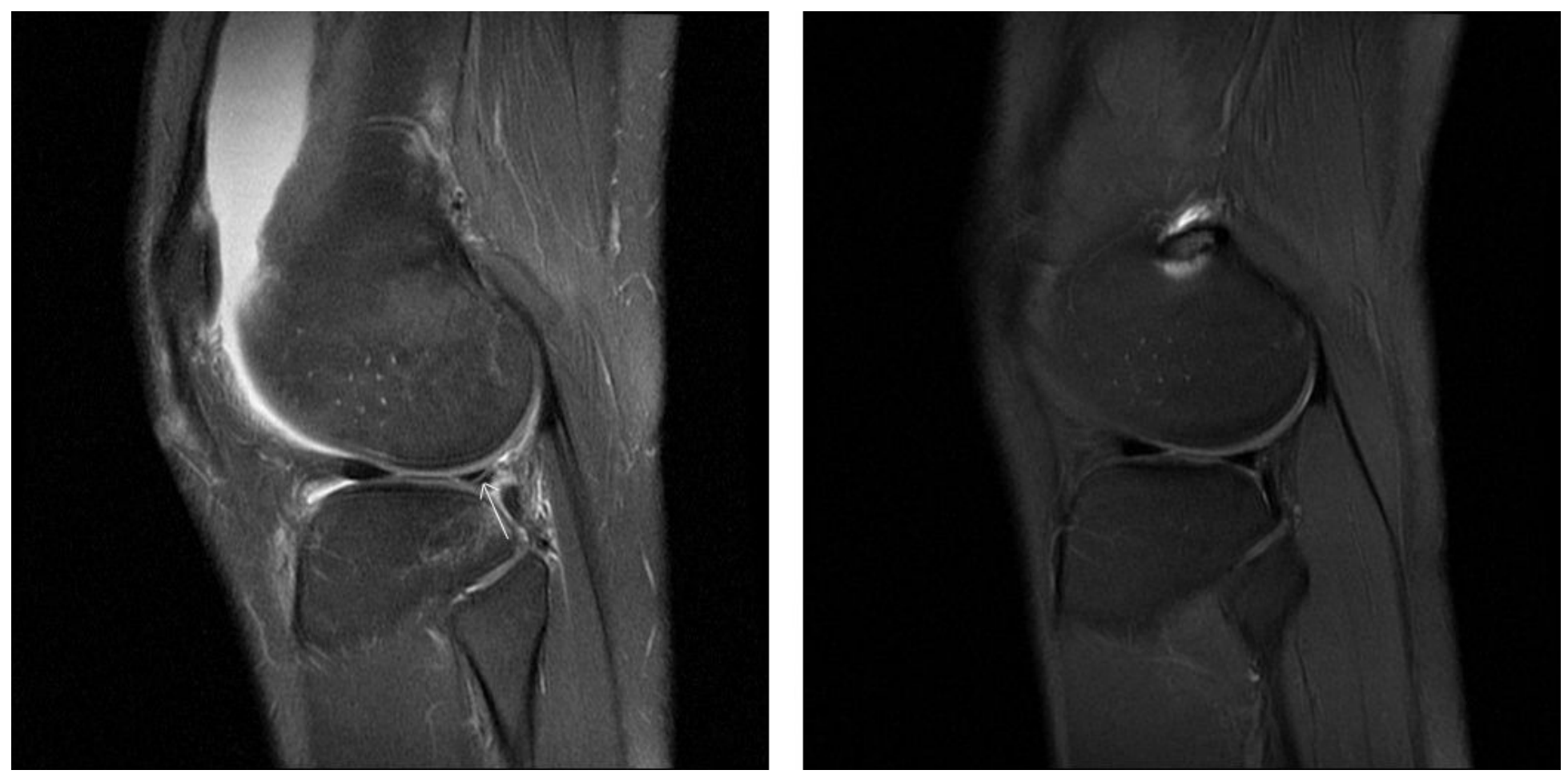

\section{Figure 3}

a Prepostoperative MRI showed a lateral meniscal tear anterior to the popliteal hiatus (white arrow). b Postoperative MRI showed that the repaired lateral meniscus obtained healing.

\section{Supplementary Files}

This is a list of supplementary files associated with this preprint. Click to download.

- data.xlsx 